\title{
Changing demographic and vital statistics patterns in the Region during the past 50 years
}

Esmat I. Hammoud'

\section{Introduction}

The objective of this article, as indicated in the title, is to review changes in demographic patterns and in vital statistics in the Eastern Mediterranean Region (EMR) of the World Health Organization (WHO) on the 50th anniversary of WHO's inauguration in 1948.

Before starting this review a number of issues have to be considered.

\section{Geographical coverage}

At the time regionalization of WHO began the EMR covered Aden, British Somaliland, Cyprus, Egypt, Eritrea, Ethiopia, French Somaliland, Greece, Iran, Iraq, Lebanon, Pakistan, Palestine, Saudi Arabia, Syria, Transjordan, Tripolitania, Turkey and Yemen.

Since then several changes have occurred with some countries transferring to or from other regions. It has been decided to follow the composition of the EMR as it stands now, since it would be impossible to readjust the data to reflect the constituent countries at each date. It should be noted that the term "country" as used here also refers at times to territories and areas.

\section{Sources and nature of data}

One problem to be resolved was finding adequate sources of data. Earlier issues of specialized UN periodic publications, particularly the Demographic yearbook and the quarterly Population and vital statistics report were not available at the Regional Office. The World health statistics annuals were available as a complete set but unfortunately very few EMR countries appeared in earlier issues.

The UN publication World population prospects $1990[I]$, the latest available when this paper was prepared, was the main reference used in this article. It gives detailed demographic and vital statistics estimates at five-year intervals starting in 1950, which are perfect for the purpose of this article. When discussing trends one has to study data at reasonably long intervals. Hence data for 1950,1965 and 1980 , three points all 15 years apart, were selected from this source.

Data for 1995 could not be selected from this source as the document was prepared in 1990. Projecting these data to 1995 would certainly not reflect the developments in the Region including the flaring up or cessation of wars and internal conflicts; the resulting massive population movements in some countries; international penalties and their health consequences in a few countries; and recent changes in national regulations dealing with expatriate labour forces and foreign settlers in general in others. Fortunately, a database is maintained in the Regional Office for the Eastern Mediterranean, mainly derived from periodic national monitoring and evaluation reports on health-for-all strate-

'Former WHO Regional Adviser, Health Statistics. 
gies. These have served as the source for circa 1995 data used in this article.

The under-5 mortality rate (U5MR), actually the probability of dying before the age of 5 years, is a rather new development and therefore not included in the main reference. However, UNICEF's annual report, The state of the world's children 1997 [2] gives estimates for 1960 and 1997 . Hence only data for these two years were included in this article.

The following points have to be noted in data interpretation:

- Vital registration is known to be incomplete in the Region. Recent data indicate that registration is believed to be complete for births in seven EMR countries, for deaths in three and for infant deaths only in three. Hence this study is based mainly on estimates prepared by the UN.

- Estimates for vital rates in World population prospects 1990 cover five years, i.e. the rates given here as for 1950, for example, are really the estimated average annual rates for the period 1950-55, and so on.

- These estimates are based on the medium variant. i.e. the estimated medium trend in fertility variants; estimates based on high, low or constant variants were not used in this article.

- The corresponding data for 1995 are for the latest available single year.

- Since World population prospects 1990 was prepared early in 1990, the two Yemens then existing (Democratic Yemen and Yemen $\Lambda$ rab Republic) were shown separately. Data representing the unified Republic of Yemen were recalculated as the wcighted average of the two parts.

- The term "least developed countries" (LDCs), as defined by the UN General Assembly, Includes from the Region: Afghanistan, Djibouti, Somalia, Sudan and Yemen. The composition of the "more developed regions" (MDRs) and the "less developed regions" (LDRs) may be found in World population prospects 1990 [I].

As the population size and structure are essentially the result of two acting forces, changes in vital rates and in concomitant migration, it was considered advisable to start here by commenting on trends in vital rates so as to be able to understand later, changes in population size and structure.

\section{Fertility trends}

The crude birth rate (CBR) in 1950 exceeded 45 per 1000 population in all but two countries in the Region. The number of countries with such a high birth rate had reduced by 1965 to 15 countries, then in 1980 to 9 and to just 5 countries in 1995 with 4 of them among the least developed countries. In fact, the CBR in 1995 was already below 35 per thousand population in 13 countries. The regional average was $48.7 \%$ in 1950 , this changed little till 1980 (when it reached $44.3 \%$ ), but dropped to $32.5 \%$ in 1995 , compared to $24.9 \%$ for the world total, $13.4 \%$ for the more developed regions (MDRs) and 27\% for the less developed regions (LDRs).

The relative drop was least marked during 1950-65; the CBR was actually higher in 1965 than in 1950 in 8 countries (including the $4 \mathrm{LDCs}$ ). The drop was generally higher during 1965 80, but most marked during 1980-95. This may be explained by the increasing implementation, from 1980 onwards, of the health-for-all strategies, in particular the maternal and child health services (including family health). Data availablc on 15 EMR countries show that the contraception prevalence rates estimated for $1990-96$ reached $\geq 35 \%$ in 8 out of 11 countries (actually $\geq 50 \%$ in 4 ). This does not include the $4 \mathrm{LDCs}$ where the rate was $<10 \%$.

To summarize, the CBR in 1995 was lower than its level in 1950 in almost all coun-

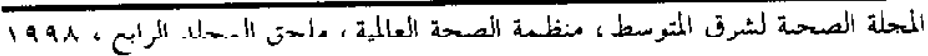


tries. It was $<50 \%$ of its 1950 level in six countries and $50 \%-75 \%$ in eight more.

The total fertility rate (TFR), which is the average number of children that would be born to a group of women of reproductive age if they experienced no mortality, is a related indicator. It has followed the same trend as the CBR though somewhat lagging behind in terms of time. This is due to the cumulative effect of high fertility that might have prevailed before.

In 1950 the TFR was $>7.0$ in nine countries and $<5.0$ in only one country. Estimates for 1965 were equal to or higher than in 1950 in 17 countries. In fact, the regional average increased from 6.76 in 1950 to 6.98 in 1965. Some drop in the TFR was seen in 1980 when it was $\geq 7.0$ in six countries but $<5.0$ in five others. A marked drop showed in 1995 when in no country did the TFR exceed 7.0 , was $<5.0$ in 14 countries and actually $<3.0$ in 4 of them. With the exception of two LDCs, the TFR in 1995 was consistently lower than in 1950 . It was $<50 \%$ of its baseline level in six countries, and $50 \%-75 \%$ of the level in nine more. The world total average was 5.00 in 1950 , then 4.89 in 1965 , dropped to 3.60 in 1980 and 3.14 in 1995 (compared to 1.90 for the MDRs and 3.47 for the LDRs).

\section{Mortality trends}

The crude death rate (CDR), which is the basic mortality indicator, behaved differently from the preceding two fertility indicators. It started to drop during 1950-65. The CDR in 1965 was consistently lower than in 1950 . in 1980 lower than in 1965, and, except in three countries, in 1995 lower than in 1980.

Thus in 1950 the CDR was $>25$ per 1000 population in 10 countries (including the five LDCs), and $<20 \%$ in only four countries. During $1950-65$ the relative drop was be- tween $5 \%$ and $46 \%$ including a drop of $>30 \%$ in eight countries. As a result, in 1965 the CDR was $<20$ per thousand in 14 countries and still $\geq 25 \%$ in only 2 countries (both among the LDCs). The drop was definitely faster during $1965-80$, being $>30 \%$ in 15 countries. In 1980 the CDR was $23.0 \%$ and $22.3 \%$ in two LDCs, otherwise $<18.5 \%$ in all other countries. It was already $<10 \%$ 。 in 12 countries.

Having reached such low figures, one would not expect the drop to continue at the same pace. The CDR in 1995 became $<10 \%$ in 16 countries, even below $5 \%$ in 6 countries. However, the relative drop from the 1980 levels was slower than during 1965-80 in 11 countries. The CDR in 1995 was actually higher than in 1980 in three countries (yet still lower than in 1965 and 1950): Afghanjstan, Iraq and Yemen for apparent reasons (the two Gulf wars and the subsequent international embargo for Iraq and internal fighting in the other two).

However, the CDR in 1995 was one-half or less of its level in 1950 in 17 countries, even one-quarter or less in 8 countries. The regional average CDR in 1950 was $26.4 \%$, then $19.6 \%$ in 1965 , then $12.8 \%$ in 1980 , reaching $9.2 \%$ in 1995 (compared to $8.6 \%$ for the world total, $9.5 \%$ or the MDRs and $8.4 \%$ for the LDRs). These trends reflect improvements in medical care and public health: control of epidemics, antibiotics, child immunization and oral rehydration. The recent rise in the CDR in the MDRs reflects the increasing proportion of elderly people in these countries.

Estimates of the infant mortality rate (IMR) for 1950 present a gloomy picture. With a range of 53-23I per 1000 live births, it was $<100$ per thousand in only two countries and $\geq 200$ per thousand in six.

The trend of the IMR exhibits a consistent drop from one reference year to the othcr, with only rare exceptions. For all 
countries, without exception, the IMR dropped during 1950-65 and 1965-80. Thus in 1965 , the range was $29 \%-203 \%$; the IMR was $>200 \%$ in one country only, but $<100 \%$ in six and $<50 \%$ in four. It was $\geq 150 \%$ in seven countries including all the five LDCs. The rates in 1965 were reduced to $11 \%-56 \%$ of their levels in 1950 .

The reduction was faster during $1965-80$, with a drop of between $10 \%-72 \%$ from the 1965 levels. In 1980, the IMR was still $\geq 100$ per thousand live births in seven countries including the five LDCs, yet < $50 \%$ in six (range 10\%-72\%). This rapid improvement continued during 1980-95 and the relative drop was $1 \%-75 \%$ of the 1980 levels. $\ln 1995$, the rate remained $\geq 100 \%$ in 5 countries (including 4 LDCs), became < $50 \%$ in 14 countries and even fell to $<925 \%$ in 8 countries. The 1995 levels were $6 \%$ $80 \%$ of their levels in 1950 and the relative drop over that period was $80 \%$ or more in

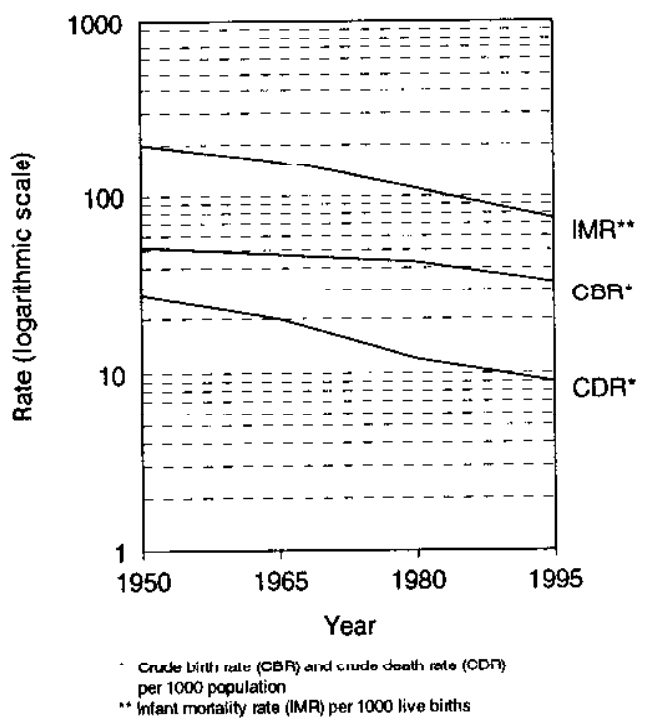

Figuro 1 Regional trends in CDR and IMR 1950-95
12 countries. The only exception to the persistent lowering trend was Iraq where the IMR in 1995 was almost $50 \%$ higher than the rate in 1980 as a result of two Gulf wars and the subsequent international embargo imposed.

The IMR regional average was 191 per 1000 live births in 1950 , then $152 \%$ in 1965 , then $106 \%$ in 1980 , declining to $75 \%$ in 1995. The corresponding averages for 1995 were $57 \%$ for the world total, $11 \%$ for the MDRs and $63 \%$ for the LDRs.

Regional trends in the CBR, CDR and IMR are shown in Figure 1.

Estinates of the under- 5 mortality rate (U5MR) in 1960 were $>200$ per 1000 live births in 17 countries and $<100 \%$ in only 2 countries; the range was $36 \%-360 \%$. The relative drop between 1960 and 1995 was so marked that the drop was $>50 \%$ of its 1960 levels in 16 countries and the remaining 6 countries included $4 \mathrm{LDCs}$. Thus the U5MR in 1995 dropped to $<50$ per 1000 in 13 countries and actually $<25 \%$ in 5 countries. In other words, the rates in 1995 were $5 \%-90 \%$ of their levels in 1960 .

The regional average rate in 1995 was $115 \%$, compared to $90 / \%$ for the world, $8 \%$ for the industrialized countries, $99 \%$ for the LDRs, and $173 \%$ for the LDCs.

\section{Trends in life longevity (Figure 2)}

One can expect a prevailing high level of mortality to be reflected in low longevity. In 1950 , for example, the life expectancy at birth (both sexes combined) was in the range of 31.6-67.0 years. It was $<40$ years in eight countries (including all the five LDCs) and $\geq 55$ years in only three countries.

Between 1950 and 1965 the absolute increase in life expectancy was 3.3-11.0 years. Life expectancy in 1965 was still 


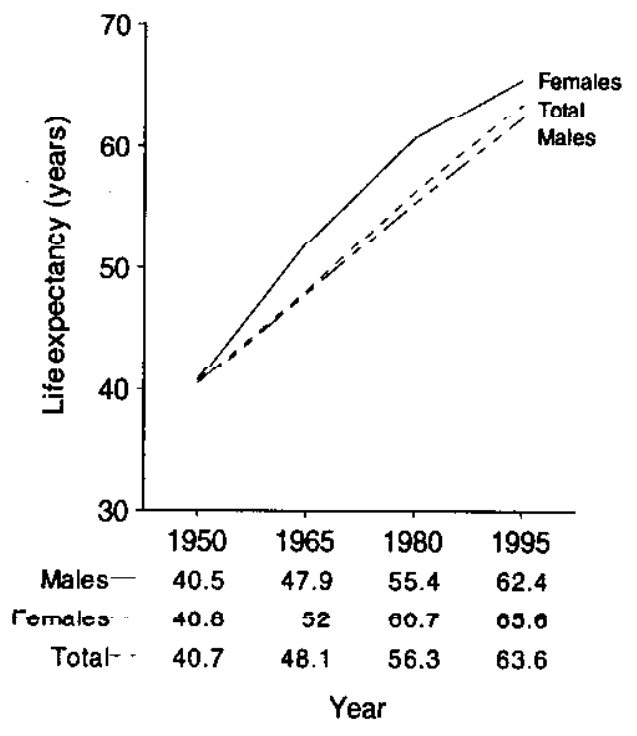

Figure 2 Trends in life expectancy by sex 1950-95

$<40$ years in three countries (all among the LDCs), $\geq 55$ years in six and $\geq 60$ years in four countries. The range was $36.0-$ 70.3 years. The absolute increase during 1965-80 was in the range of 2.1-14.2 years; in fact the increase during 1965-80 was the highest among the three periods in 11 countries. Thus, by 1980 , no country had a life expectancy of < 40 years; it was $\geq 55$ years in 16 and even $\geq 60$ in 12 countries (range 40.5-75.0 years). Over the period of 30 years (1950-80) life expectancy increased by 15 years (i.e. an average of 0.5 years or more per year) in 14 countries.

With continuing reductions in mortality, though at a lower rate, life expectancy continued to rise, also at a slower rate. By 1995 , life expectancy was $<60$ years in only six countries (including all the Region's LDCs) and already $\geq 70$ years in eight countries. It was only in Iraq that the life expectancy dropped between 1980 and 1995, for the reasons mentioned earlier affecting mainly infants and young children.
In other words, life expectancy over the 45 years reviewed increased by some $10.6-35.2$ years. The increase was $<20$ years in eight countries including four of the LDCs, three where it started relatively high in 1950 ( $\geq 55$ years), and in Iraq. On the other hand, the increase reached 22.5 years or more (an average of 0.5 years per year) in half of the countries of the Region.

The regional average life expectancy was only 40.7 years in 1950 , then 48.1 in 1965 , then 56.3 in 1980 reaching 67.0 years in 1995 (compared to 67.0 years for the world, 75.8 for the MDRs and 65.0 for the LDRs).

By sex, life expectancy followed the normal pattern. With very few exceptions (mainly in the earlier periods in three countries), it had always been longer anuong females. Thus in 1950 life expectancy was $\geq 55$ years for females in three countries and for males in one country only. In 1965 it was $\geq 60$ years for females in six and for males in three countries. In 1980 it was $>65$ years for $\mathrm{fe}$ males in seven but for males in five countries. By 1995 it was $\geq 70$ in 11 countries for females against only 5 countries for males. The regional average was 65.6 years for females against 62.4 years for males.

The absolute increase during the intervals was also generally higher among females. Thus during 1950-65 and $1965-80$, the absolute increase in years was higher among females than among males in 33 out of 44 readings. Over the whole period 1950-95, the increase was higher among females in 15 countries, indicating a widening gap in life expectancy between the two sexes. The increase in life expectancy over the 45 years reached an annual average of 0.5 years among females in 13 countries and among males in only 10 countries. In 1950 , the extent of greater longevity among females than males was $>3.0$ years in 5 countries against 12 countries in 1995. 


\section{Demographic trends}

The total population size for the Region in 1950 was only 131.0 million, a little below the population of Pakistan alone in 1995. The range in population size of the countries was very wide ( $25000-40$ million). The mean population size was 6.0 million per country, while the median was only 2.8 million, due to the small population of many countries. It was $<200000$ in five countries and $>15$ million in three.

By 1965 the total population rose to 188.7 million, or an average increase of 3.8 million per year. The regional population became $144 \%$ of its size in 1950 (range $118 \%-310 \%$ ). This trend has continued up to 1995. Thus the total population reached 284.1 million in 1980 (an average increase of 6.4 million per year), then 433.2 million in 1995 (an average increase of 9.9 million per year). At each point the population was

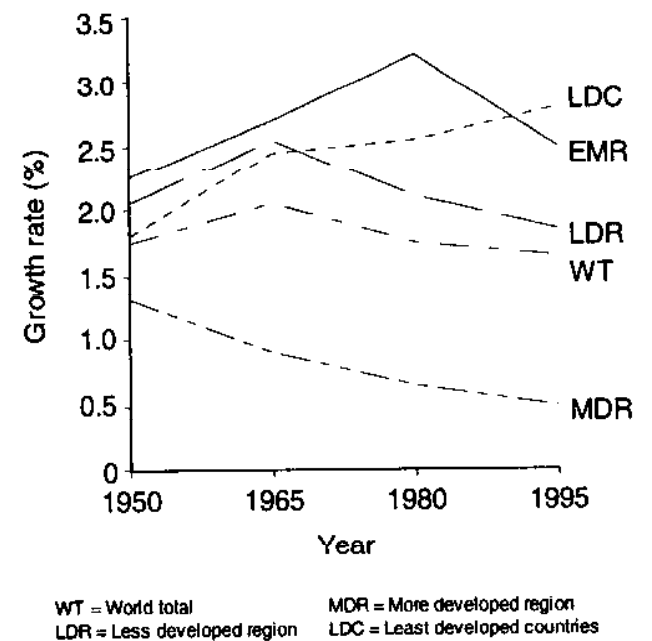

Figure 3 Trends in population growth rate in tho world $1950-95$ about $152 \%$ of that of the preceding point (range $108 \%-705 \%$ ). As a whole, the regional population in 1995 was $>330 \%$ of its size in 1950 . But the ratio varjes a great deal between the countries, with a range from just 1.5 times up to 34 times the population size in 1950 .

Trends in the population growth rate (FigIre 3) reflect trends in vital statistics and in international migration. In 1950, for example, the CBR and CDR were both high, limiting natural increase. Hence the annual growth rate was $1.5 \%-2.5 \%$ in the majority of countries (range $1.41 \%-6.73 \%$, regional average $2.25 \%$ ). It was $<2.0 \%$ in eight countries but $\geq 3.0 \%$ in only three).

The CDR started to drop during 1950-65 and by a greater extent than the CBR. plus immigration in some countries increased. The result was sizeable population growth. In fact the growth rate rose between 1950 and 1965 in 18 countries. Unlike in 1950 , the rate was $<2.0 \%$ in two countries only, became $\geq 3.0 \%$ in nine and exceeded $5.0 \%$ in four countries.

Population growth continued during 1965-80 due to a greater drop in CDR than in CBR. By 1980 those with a growth rate $\geq$ $3.0 \%$ increased to 16 countries, but in 8 countries it was lower than in 1965. For the first, and only, time we had a negative population growth in 1980 in two countries, Afghanistan and Lebanon, for known reasons. With the increasing drop in the CRR during 1980-95, population growth started to slow down. The growth rate in 1995 was lower than in 1980 in 15 countries, was $\geq 3.0 \%$ in 11 countries only, but became $<2.0 \%$ in 5 countries.

The regional average population growth rate in 1950 was $2.25 \%$, rose to $2.71 \%$ in 1965 , then to $3.20 \%$ in 1980 , but dropped to $2.60 \%$ in 1995 (compared to $1.63 \%$ for the world total, $0.45 \%$ for the MDRs, $1.94 \%$ for the LDRs, and as high as $2.83 \%$ for the $\mathrm{LDCs}$ ).

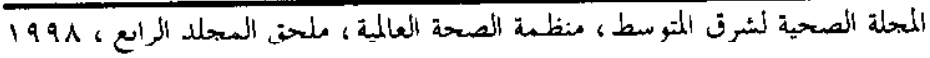




\section{Population composition}

The population proportion $<5$ years of age is essentially a function of the level of fertility. In 1950 the proportion was $<15 \%$ in only Cyprus and Lebanon; both had the lowest fertility and longest life expectancy. At the same time the proportion was $\geq 18 \%$ in four countries.

It was mentioned earlier that the TFR increased between 1950 and 1965 (regional average from 6.76 to 6.98 ). As a result, the proportion $<5$ years of age was higher in 1965 than in 1950 in 17 countries, becoming $\geq 18 \%$ in 11 countries. The proportion started declining during 1965-80, being lower in 1980 than in 1965 in 13 countries, and was $<15 \%$ in 4 countries. With the big reduction in fertility during 1980-95. the proportion $<5$ years of age dropped in 19 countries by 1995 , reaching $<15 \%$ in 15 countries. The regional average proportion was $16.6 \%$ in 1950 , rose to $18.1 \%$ in 1965 , and dropped to $17.0 \%$ in 1980 then to $13.0 \%$ in 1995 (compared to a world total average of $11.7 \%$, with $6.8 \%$ for the MDRs and $13.0 \%$ for the LDRs).

One should remember that in the case of new settlers, or with immigrants from neighbouring countries, the whole family moves. This partly explains the high proportion of children $<5$ in some countries, such as Djibouti, Pakistan, Somalia and Sudan. This is different from selective expatriate labour forces, which mainly comprised adult males, for example in Iraq, Libyan Arab Jamahiriya and the member countries of the Gulf Cooperation Council.

The proportion $<15$ years of age follows a similar trend to the $<5$. It tended to rise during 1950-65, and to fall thereafter. In 1950 , for example, the proportion was $<40 \%$ in seven countries and $245 \%$ in three. The proportion increased during $1950-65$ in 17 countries, to become $<40 \%$ in four and $\geq 45 \%$ in seven countries. But it started to drop thereafter so that, in 1995 , it was $<40 \%$ in 11 countries and $\geq 45 \%$ in only 2 countries. The regional average rose from $40.6 \%$ in 1950 to $44.2 \%$ in 1965 , but came down to $43.6 \%$ in 1980 then to $41.0 \%$ in 1995 (compared to $32.0 \%$ for the worid total, $20.7 \%$ for the MDRs and $35.1 \%$ for the LDRs).

Lowering trends in fertility and child mortality take a long time to result in an aging of the population. The population proportion $\geq 65$ years, being generally small, does not show any definite trends. In 1950 , for example, it was $<2.5 \%$ in one country only, and $2.5 \%-3.5 \%$ in 12 countries. The number of countries with a proportion $<2.5 \%$ progressively increased to reach seven countries in 1995 , but those with a proportion $2.5 \%-3.0 \%$ fluctuated to end in five countries. The regional average was $4.0 \%$ in 1995 (compared to $6.5 \%$ for the world total, $13.0 \%$ for the MDRs and $4.7 \%$ for the LDRs).

Evidently, with development and urbanization, the proportion of the urban population showed a continuous rise between 1950 and 1995 , with a wide variation between the countries. In 1950, for example, the range was $1.9 \%-62.9 \%$, with a regional average of $21.3 \%$. The urban population increased to $46.0 \%$ in 1995 (compared to a world total average of $48.1 \%$, for MDRs $73.6 \%$, and for LDRs $41.2 \%$ ). Unfortunately, the definition of "urhan" varies widely between countries, and it is also likely that the definition in the same country may change over time.

\section{Summary}

1. The CDR started to decline early, thanks to technology transfer (control of epidemics, antibiotics, immunization, oral rehydration therapy, improved medical care, etc.). 
2. The IMR showed a similar trend. Unexpectedly, however, the pace of decline was soinewhat less marked than the decline in the CDR, since infant deaths are mostly due to controllable external factors. For the world total, for example, the CDR in 1995 was $44 \%$ of its level in 1950 , and the corresponding figure for the IMR was $37 \%$. This may be due to the unfavourable conditions in the LDCs.

3. The CBR showed a marginal decline of $9 \%$ between 1950 and 1980 , but a drop of $27 \%$ between 1980 and 1995 , as a result of a drop of $29 \%$ in the IMR during the same period, implementation of healthfor-all strategies, and promotion of family health and use of contraceptives. In other words, the drop in the CBR was delayed behind and was less marked than the drop in the CDR.

4. The TFR showed an initial rise between 1950 and 1965 , then dropped thereafter from 6.98 in 1965 to 4.70 in 1995.

5 . The rate of population growth rose progressively from $2.25 \%$ in 1950 to $3.20 \%$ in 1980 , thereafter dropped to $2.60 \%$ by 1995. The regional population more than tripled $(330 \%)$ between 1950 and 1995 .
6. The drop in mortality caused an increase in life expectancy, with an average annual increase in longevity of a little above 0.5 years per year, with the increase being higher among females.

7. Changes in the CBR and CDR take a long time to be reflected in the age/sex structure of the population. The proportion $<5$ ycars, after an initial rise, dropped from $18.1 \%$ in 1965 to $13.0 \%$ in 1995 and the proportion aged $<15$ from $44.2 \%$ to $41.0 \%$. The elderly population increased slightly from $3.5 \%$ to $4.0 \%$. This implies an increase in the adult (15-64 years) population. The proportion of women in the child bearing age (15-49 years) increased slightly from $21.5 \%$ in 1965 to $23.0 \%$ in 1995 .

8. The proportion of urban population increased progressively from $21 \%$ to $46 \%$. This rather rapid increase in urbanization, if accompanied by poverty, low education, insufficient diet and overcrowding (as in the LDCs), may strain the capacity of environmental health facilities and services, leading to degradation of living conditions and, subsequently, of health status.

\section{References}

1. World population prospects 1990 . New York, United Nations, Department of International and Economic Social Affairs, 1991.
2. United Nations Children's Fund. The state of the world's children 1997. New York, Oxford University Press, 1997. 